\title{
Hepatic encephalopathy induced by Lenvatinib and anti-PD-1 mAb in a patient with advanced hepatocellular carcinoma: A case report
}

\author{
ZHEN LIU $^{1 *}$, YING MENG $^{1 *}$, WENMING XIAO $^{2}$, HUIJUN WANG $^{1}$, \\ LI SUN $^{1}$, ZHENG FU $^{1}$, XUEJIE XIONG ${ }^{3}$ and SHU ZHANG ${ }^{1}$ \\ ${ }^{1}$ Department of Gastroenterology Oncology, Shandong Cancer Hospital and Institute, Shandong First Medical University \\ and Shandong Academy of Medical Sciences, Jinan, Shandong 250117; ${ }^{2}$ Department of Clinical Oncology, \\ Taian City Central Hospital, Taian, Shandong 271000; ${ }^{3}$ Department of Oncology, \\ Xiamen Changgung Hospital, Xiamen, Fujian 361000, P.R. China
}

Received September 28, 2020; Accepted March 19, 2021

DOI: $10.3892 / \mathrm{mco} .2021 .2272$

\begin{abstract}
Previous studies have reported that the combinational therapy of Lenvatinib and anti-programmed cell death-1 (PD-1) monoclonal antibody $(\mathrm{mAb})$ produced a longer overall survival in patients with hepatocellular carcinoma (HCC). The current case report presented a a patient with HCC who had hepatic encephalopathy (HE) following treatment with Lenvatinib and anti-PD-1 mAb. The 42-year-old patient was diagnosed with stage IVa HCC accompanied with cirrhosis and Child-Pugh C. Computed tomography (CT) imaging revealed collateral circulation of the portal vein, causing significant varicose veins in the gastric fundus, mesenteric varices and colon edema. The patient was administered $12 \mathrm{mg}$ Lenvatinib once daily combined with $240 \mathrm{mg}$ anti-PD-1 mAb. After 3 days of treatment, he presented with a disorder of psychoneurosis and blood ammonia (248 $\mu \mathrm{g} / \mathrm{dl}$; normal levels, 40-80 $\mu \mathrm{g} / \mathrm{dl})$. A cranial CT scan exhibited no significant abnormalities. The patient rapidly progressed from grade 1 to grade $3 \mathrm{HE}$. Lenvatinib treatment was discontinued. After admission to the intensive care unit, the patient's blood ammonia level dropped to $132 \mu \mathrm{g} / \mathrm{dl}$, after which he was discharged. It was concluded that the portal vein collateral circulation in the patient with HCC may have caused HE development whilst receiving Lenvatinib and anti-PD-1 $\mathrm{mAb}$ combinational therapy.
\end{abstract}

Correspondence to: Dr Shu Zhang, Department of Gastroenterology Oncology, Shandong Cancer Hospital and Institute, Shandong First Medical University and Shandong Academy of Medical Sciences, Jiyan Road No. 440, Jinan, Shandong 250117, P.R.China

E-mail: zhangshu@csco.org.cn

*Contributed equally

Key words: anti-programmed cell death-1 monoclonal antibody, case report, hepatic encephalopathy, hepatocellular carcinoma, Lenvatinib

\section{Introduction}

Liver cancer is the third leading cause of cancer death in the world, with approximately 745 thousand deaths every year (1). Hepatocellular carcinoma (HCC) is the most common pathological type of primary liver cancer in adults. It usually occurs in patients with chronic liver disease, especially those with cirrhosis. Most patients with HCC were frequently diagnosed late and had lost the opportunity of resection at the time of diagnosis. Compared with placebo, first-line sorafenib therapy prolonged overall survival (OS) of patients with advanced HCC by 2.8 months (2). Recently, the REFLECT study showed that lenvatinib was non-inferior to sorafenib in terms of OS. Although this study reported serious adverse effects caused by lenvatinib, such as liver failure, cerebral hemorrhage, and respiratory failure, no adverse events of hepatic encephalopathy (HE) were highlighted (3). The combination of lenvatinib and anti-programmed cell death-1 (PD-1) monoclonal antibody $(\mathrm{mAb})$ has been explored in clinical practice. For example, a phase III study showed that the combination of lenvatinib with anti-PD-1 mAb led to a 17-month OS in patients with advanced HCC (4). However, whether the combined therapy increased the HE risk remained unclear. This study reported a case of clinical HE occurrence in a patient with HCC receiving the combination therapy of lenvatinib and anti-PD-1 mAb.

\section{Case report}

A 42-year-old male patient had abdominal discomfort in September 2018, with no other symptoms such as nausea, vomiting, melena, or back pain. Computed tomography (CT) of the abdomen performed in a hospital in Beijing revealed a large hepatic tumor; ascites; thrombi of the portal vein, hepatic vein, and inferior vena cava; and varicose veins in the lower esophagus, around the gastric fundus, and in the splenic hilum. He was diagnosed with advanced HCC. Sorafenib $400 \mathrm{mg}$ twice daily was administered for 6 months. In March 2019, his CT images examined at Taian Hospital 
Table I. General characteristics of the patient.

\begin{tabular}{lc}
\hline Characteristic & Value \\
\hline Age (years) & 42 \\
Sex & Male \\
Diagnosis & Hepatocellular carcinoma \\
Stage & IVa \\
Child-Pugh & $\mathrm{C}$ \\
BCLC stage & $\mathrm{D}$
\end{tabular}

Present

Tumor thrombus

Portal vein

Hepatic vein

Inferior vena cava

Varicose veins

Lower esophagus

Gastric fundus

Splenic hilum

First-line therapy

Second-line therapy

WBC count $\left(\times 10^{9} / 1\right)$

Before treatment

After treatment

Normal range

$\mathrm{HB}(\mathrm{g} / \mathrm{l})$

Before treatment

After treatment

Normal range

PLT (x10 $\%$ l)

Before treatment

After treatment

Normal range

ALT (U/l)

Before treatment

After treatment

Normal range

AST (U/l)

Before treatment

After treatment

Normal range

TBIL $(\mu \mathrm{mol} / \mathrm{l})$

Before treatment

Present

Present

Present

Present

Present

Present

$400 \mathrm{mg}$ bid Sorafenib

$12 \mathrm{mg}$ qd Lenvatinib + $240 \mathrm{mg}$ qd Nivolumab

10.52

11.33

4-10

107

118

Male, 120-160; Female, 110-150

After treatment

Normal range

DBIL $(\mu \mathrm{mol} / \mathrm{l})$

Before treatment

After treatment

Normal range

IBIL $(\mu \mathrm{mol} / \mathrm{l})$

Before treatment

After treatment

Normal range
Table I. Continued.

\begin{tabular}{lc}
\hline Characteristic & Value \\
\hline Alb (g/l) & \\
Before treatment & 28.5 \\
After treatment & 7.5 \\
Normal range & $35-51$ \\
BUN (mmol/l) & \\
Before treatment & 3 \\
After treatment & 3.9 \\
Normal range & $2.86-7.14$ \\
SCr $(\mu$ mol/l) & \\
Before treatment & 36 \\
After treatment & 39 \\
Normal range & $44-133$ \\
NH3 $(\mu \mathrm{g} / \mathrm{dl})$ & \\
Before treatment & 0 \\
After treatment & 248 \\
Normal range & $40-80$
\end{tabular}

BCLC, Barcelona Clinic Liver Cancer; WBC, white blood cell; HB, hemoglobin; PLT, -platelet; ALT, alanine transaminase; AST, aspartate aminotransferase; TBIL, total bilirubin; DBIL, direct bilirubin; IBIL, indirect bilirubin; Alb, albumin; BUN, blood urea nitrogen; $\mathrm{SCr}$, serum creatinine.

showed multiple nodules in the left hepatic lobe and hilar area. The largest one was measured as about $10.7 \times 7.2 \mathrm{~cm}^{2}$, the right branch of the portal vein was compressed and thinned, and some solid masses were seen in the left branch. Portal vein tumor thrombus and moderate ascites were observed (Fig. 1). The laboratory results were as follows: alanine aminotransferase (ALT), $29 \mathrm{U} / 1$; aspartate aminotransferase (AST), $77 \mathrm{U} / 1$; total bilirubin (TBIL), $110.2 \mu \mathrm{mol} / \mathrm{l}$; direct bilirubin (DBIL), $92.5 \mu \mathrm{mol} / 1$; indirect bilirubin (IBIL), $17.7 \mu \mathrm{mol} / \mathrm{l}$; albumin (ALB), $25.7 \mathrm{~g} / \mathrm{l}$; prothrombin time (PT), $16.8 \mathrm{~s}$; and alpha-fetoprotein (AFP), $127.7 \mathrm{ng} / \mathrm{ml}$. The patient was defined as stage IVa (cT4N1M0), Child-Pugh grade C, and BCLC stage D (Table I). Glutathione and polyphosphatidylcholine were added as symptomatic treatments. On April 12, 2019, the liver function was tested again, and the results were as follows: ALT, $36 \mathrm{U} / 1$; AST, $73 \mathrm{U} / 1$; TBIL, $139 \mu \mathrm{mol} / 1$; DBIL, $106.5 \mu \mathrm{mol} / 1$; IBIL, $32.8 \mu \mathrm{mol} / 1$; and ALB, $28.5 \mathrm{~g} / \mathrm{l}$. The patient's condition got worse. On April 13, 2019, the treatment of lenvatinib $12 \mathrm{mg}$ once daily combined with anti-PD-1 mAb $240 \mathrm{mg}$ was started. On the third day after the medication, the patient developed emotional abnormalities and mild cognitive impairment and was not able to identify anyone. On the fourth day, the patient developed a disorder of consciousness and slurred speech. The patient did not cooperate with the physical examination and was stunned, blurred, poor in spirit, and irritable with mixed aphasia. The pupil diameter increased to $4 \mathrm{~mm}$ on both sides, and it was slow to respond to light. Physical examination results were muscle strength grade 5, normal muscle tension, bilateral palmomental reflex $(+)$, tendon hyperreflexia $(++)$, Babinski 


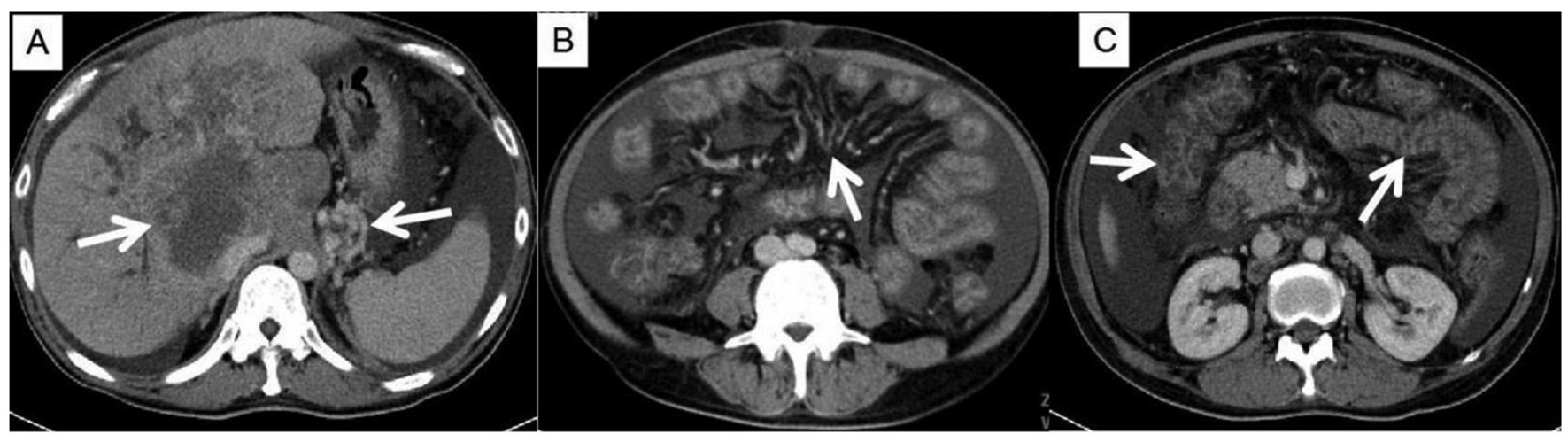

Figure 1. Computed tomography (CT) scan revealed multiple metastases in the liver with poorly defined borders, (A) irregular morphology (left white arrow), marked gastric varices (right white arrow), (B) mesenteric varices (white arrow), (C) moderate colon edema (left white arrow) and mild intestinal edema (right white arrow).

sign positive, and ataxia. A cranial CT scan showed no significant abnormalities. The laboratory results were as follows: blood ALT, $48 \mathrm{U} / \mathrm{l}$; AST, $98 \mathrm{U} / \mathrm{l}$; TBIL, $90 \mu \mathrm{mol} / \mathrm{l}$; DBIL, $82.5 \mu \mathrm{mol} / 1$; IBIL, $7.5 \mu \mathrm{mol} / 1$; ALB, $34.4 \mathrm{~g} / 1$; and blood ammonia, $248 \mu \mathrm{g} / \mathrm{dl}$. The patient was diagnosed as grade 3 HE. Lenvatinib was discontinued, and the patient was transferred to the intensive care unit for symptomatic treatment, including oxygen inhalation, sedation, fluid replacement, correction of electrolyte disturbances, liver protection, and enema. The ornithine aspartate injection was also used to reduce the blood ammonia level. On the fifth day, the patient's condition, including consciousness, spirit, and sleep, greatly improved, and his irritability disappeared. The blood test revealed the following: ALT, $39 \mathrm{U} / 1$; AST, $86 \mathrm{U} / 1$; TBIL, $126.2 \mu \mathrm{mol} / 1$; DBIL, $95.7 \mu \mathrm{mol} / 1$; IBIL, $30.5 \mu \mathrm{mol} / 1$; ALB, $28.6 \mathrm{~g} / \mathrm{l}$; and blood ammonia, $132 \mu \mathrm{g} / \mathrm{dl}$. Finally, the patient was discharged due to his improved physical status.

\section{Discussion}

The REFLECT study was an open-label, multicenter, non-inferiority phase III clinical study to compare the efficacy and safety of lenvatinib versus sorafenib in the first-line treatment of patients with unresectable advanced HCC) (3). It revealed that the median survival time of lenvatinib was 13.6 months, which was non-inferior to that of sorafenib (12.3 months). The study showed that the most common any-grade adverse events associated with the use of lenvatinib included hypertension (42\%), diarrhea (39\%), decreased appetite (34\%), and weight loss $(31 \%)$. Fatal adverse events occurred in $11(2 \%)$ patients, including liver failure (3 patients), cerebral hemorrhage ( 3 patients), and respiratory failure ( 2 patients). The adverse events associated with the use of sorafenib included hand-foot syndrome, diarrhea, hypertension, and decreased appetite. No adverse events of $\mathrm{HE}$ were reported in the REFLECT study.

$\mathrm{HE}$ is a serious complication of severe liver disease. It is mainly manifested as a spectrum of neuropsychiatric abnormalities in patients with liver dysfunction, after exclusion of brain disease, and characterized by personality changes, intellectual impairment, and a depressed level of consciousness. The inducing factors mainly included severe liver disease, extensive portosystemic shunt, infection, upper gastrointestinal hemorrhage, and massive drainage of ascites. HE caused by anti-PD-1 mAb or a combination of anti-PD-1 mAb and lenvatinib in $\mathrm{HCC}$ has not been reported so far.

Namba et al, (5) reported a case of lenvatinib monotherapy-induced HE in HCC. The patient was diagnosed with HCC in 2016 and received hepatic lobectomy. His CT showed a portosystemic shunt between the superior mesenteric vein and the right testicular vein. In July 2018, the reexamination of $\mathrm{CT}$ and laboratory parameters showed multiple lung metastases and lymph node metastases, with the blood ammonia level of $39 \mu \mathrm{mol} / \mathrm{l}$; the patient was reviewed as Child-Pugh A grade. The patient was treated with lenvatinib after progression. Five days after taking lenvatinib (12 mg/day), the patient developed grade $3 \mathrm{HE}$ (type B). Lenvatinib was discontinued after the eighth day of medication due to no significant improvement in the condition after the corresponding therapy, and then the symptoms were relieved. On the fourth day of discontinuation, lenvatinib was restarted with a reduced dose ( $8 \mathrm{mg} / \mathrm{day})$. Grade $2 \mathrm{HE}$ and elevated blood ammonia level (145 $\mu \mathrm{mol} / \mathrm{l})$ appeared again, and lenvatinib was discontinued again. A surgery was performed to block the collateral circulation of the portal system, and then lenvatinib was re-administered (12 $\mathrm{mg} / \mathrm{day}$ ) successfully without the recurrence of HE. It was concluded that lenvatinib might induce $\mathrm{HE}$ by increasing the portal collateral circulation of the hepatic portal vein.

In addition, a multicenter, open-label phase I clinical trial on the use of lenvatinib in ttreating advanced HCC enrolled 20 patients. Two patients had grade $3 \mathrm{HE}$ : one with Child-Pugh A and the other with Child-Pugh B. They took lenvatinib at doses of 16 and $12 \mathrm{mg} / \mathrm{day}$, respectively. It was presumed that high doses of lenvatinib might induce HE (6).

This novel study reported a case of HE induced by lenvatinib combined with anti-PD-1 mAb in treating advanced HCC with Child-Pugh C. This patient had advanced HCC with cirrhosis, and the CT image showed a collateral circulation of the portal vein, causing significant varicose veins in the gastric fundus (Fig. 1A), mesenteric varices (Fig. 1B), and edema of colon and small intestine (Fig. 1C). On the third day of the combination treatment, the patient 
developed grade $1 \mathrm{HE}$ and rapidly progressed to grade $3 \mathrm{HE}$. After lenvatinib was discontinued, the blood ammonia level decreased and symptoms relieved. In this case, HE occurred after the treatment of lenvatinib combined with anti-PD-1. This patient presented Child-Pugh $\mathrm{C}$ and had a very high bilirubin level, which were common manifestations in advanced HCC and had a trend to present with HE. However, the patient did not display key clinical characteristics for the diagnosis of HE, including abnormal blood ammonia level, before the combined treatment of lenvatinib with anti-PD-1 $\mathrm{mAb}$. After a few days of this combination treatment, he again presented with $\mathrm{HE}$. Which drug induced $\mathrm{HE}$ in this patient with Child-Pugh $\mathrm{C}$ was not known. Based on the aforementioned findings, it was believed that HE was mainly induced by lenvatinib in this case. HE has many causative factors, such as the portal-systemic venous shunt. This case illustrated that the formation of collateral circulation in the portal vein might be the basis of lenvatinib-induced $\mathrm{HE}$. Therefore, when treating patients with HCC having a poor liver function, such as cirrhosis and portal vein collateral circulation, whether to use lenvatinib, what dose to use, and under what conditions to choose anti-PD-1 monoclonal antibody combination therapy need to be addressed in the future.

In conclusion, the patient with $\mathrm{HCC}$ showing a portal vein collateral circulation on $\mathrm{CT} / \mathrm{magnetic}$ resonance imaging images might have a high risk of developing $\mathrm{HE}$ after treatment with the combination therapy of lenvatinib and anti-PD-1.

\section{Acknowledgements}

Not applicable.

\section{Funding}

The present study was supported by funds from the Shandong Medical and Health Science and Technology Development Plan Project (grant no. 2017WS266) and the Scientific Research Project of the Shandong Academy of Medical Sciences (grant nos. 201730, 201808 and 201838).

\section{Availability of data and materials}

All data generated or analyzed during this study are included in this published article.

\section{Authors' contributions}

$\mathrm{ZL}$ and SZ carried out the case report, collected the data and drafted the manuscript. ZL, WX, ZF and SZ performed statistical analysis and participated the study design. ZL, HW, LS, YM and XX participated in the acquisition, analysis and interpretation of data, and drafted the manuscript. All authors read and approved the final manuscript. ZL and SZ confirm the authenticity of all the raw data.

\section{Ethics approval and consent to participate}

The patient provided written informed consent.

\section{Patient consent for publication}

Written informed consent was obtained from the patient for publication of this case report and any accompanying images.

\section{Competing interests}

The authors declare that they have no competing interests.

\section{References}

1. Ferlay J, Soerjomataram I, Dikshit R, Eser S, Mathers C, Rebelo M, Parkin DM, Forman D and Bray F: Cancer incidence and mortality worldwide: Sources, methods and major patterns in GLOBOCAN 2012. Int J Can 136: E359-386, 2015.

2. Llovet JM, Ricci S, Mazzaferro V, Hilgard P, Gane E, Blanc JF, de Oliveira AC, Santoro A, Raoul JL, Forner A, et al: Sorafenib in advanced hepatocellular carcinoma. N Engl J Med 359: 378-390, 2008.

3. Kudo M, Finn RS, Qin S, Han KH, Ikeda K, Piscaglia F, Baron A, Park JW, Han G, Jassem J, et al: Lenvatinib versus sorafenib in first-line treatment of patients with unresectable hepatocellular carcinoma: A randomised phase 3 non-inferiority trial. Lancet 391: 1163-1173, 2018.

4. Yamashita T, Kudo M, Ikeda K, Izumi N, Tateishi R, Ikeda M, Aikata H, Kawaguchi Y, Wada Y, Numata K, et al: REFLECT-a phase 3 trial comparing efficacy and safety of lenvatinib to sorafenib for the treatment of unresectable hepatocellular carcinoma: An analysis of Japanese subset. J Gastroenterol 55: 113-122, 2020.

5. Namba M, Kawaoka T, Aikata H, Kodama K, Uchikawa S, Ohya K, Morio K, Fujino H, Nakahara T, Murakami E, et al: Percutaneous transvenous shunt occlusion for portosystemic encephalopathy due to lenvatinib administration to a patient with hepatocellular carcinoma and portosystemic shunt. Clin J Gastroenterol 12: 341-346, 2019.

6. Ikeda M, Okusaka T, Mitsunaga S, Ueno H, Tamai T, Suzuki T, Hayato S, Kadowaki T, Okita K and Kumada H: Safety and pharmacokinetics of lenvatinib in patients with advanced hepatocellular carcinoma. Clin Cancer Res 22: 1385-1394, 2016. 